\title{
Analytical Solutions of Fractional Differential Equations Using the Convenient Adomian Series
}

\author{
Xiang-Chao Shi, ${ }^{1,2}$ Lan-Lan Huang, ${ }^{3}$ Zhen-Guo Deng, ${ }^{4}$ and Dan Liu ${ }^{3}$ \\ ${ }^{1}$ State Key Laboratory of Oil and Gas Reservoirs Geology and Exploration, Southwest Petroleum University, Chengdu 610500, China \\ ${ }^{2}$ State Key Laboratory of Hydraulics and Mountain River Engineering, College of Water Resources and Hydropower, \\ Sichuan University, Chengdu 610500, China \\ ${ }^{3}$ Key Laboratory of Numerical Simulation of Sichuan Province and College of Mathematics and Information Science, \\ Neijiang Normal University, Neijiang 641112, China \\ ${ }^{4}$ School of Mathematics and Information Science, Guangxi University, Nanning 530004, China
}

Correspondence should be addressed to Lan-Lan Huang; mathlan@126.com

Received 6 April 2014; Revised 6 May 2014; Accepted 6 May 2014; Published 19 May 2014

Academic Editor: Dumitru Baleanu

Copyright (C) 2014 Xiang-Chao Shi et al. This is an open access article distributed under the Creative Commons Attribution License, which permits unrestricted use, distribution, and reproduction in any medium, provided the original work is properly cited.

Due to the memory trait of the fractional calculus, numerical or analytical solution of higher order becomes very difficult even impossible to obtain in real engineering problems. Recently, a new and convenient way was suggested to calculate the Adomian series and the higher order approximation was realized. In this paper, the Adomian decomposition method is applied to nonlinear fractional differential equation and the error analysis is given which shows the convenience.

\section{Introduction}

The fractional calculus has frequently appeared in various applied areas and has become an increasing interesting topic in the past decades [1-7]. Many efforts to analytical and numerical methods have been made. The often used numerical methods are the fractional difference method [8$10]$ and the predictor corrector method [11-13] and others as well as the analytical methods such as the variational iteration method (VIM) [14-17] and the Adomian decomposition method (ADM) [17-21] and others.

In fact, these methods are developed from original versions for ordinary differential equation of the integer order equation. Compared with the ordinary calculus, the fractional calculus has the long interaction traits or the socalled memory effects; this characteristic can better depict various nonlinear dynamics in both theories and engineering mathematical modeling. However, it also results in finding the solution to the fractional models. The challenge is analyzed in the analytical methods in [17].

The ADM has been extensively applied to fractional differential equations due to its convenience. The Adomian series should be calculated in each iteration which greatly affects the efficiency and accuracy of the analytical approximation. In order to solve this problem, Duan very recently suggested a new way to calculate the Adomian series in [2225 ] and successfully extended it to the fractional differential equation [26].

In this paper, we adopt Duan's way to calculate the Adomian series and apply it to FDEs for $1<\alpha<2$. We define the residual function and give the error analysis and investigate the validness of the iteration formulae.

\section{Algorithm of the Fractional Differential Equations}

Definition 1 (see [2]). The Caputo derivative is defined as

$$
\begin{array}{r}
{ }_{0}^{C} D_{t}^{\alpha} u=\frac{1}{\Gamma(m-\alpha)} \int_{0}^{t} \frac{1}{(t-\tau)^{\alpha-m+1}} \frac{d^{m}}{d \tau^{m}} u(\tau) d \tau, \\
0<t, 0<\alpha, m=[\alpha]+1,
\end{array}
$$

where $\Gamma$ is the Gamma function. 
Definition 2 (see [2]). The R-L integration of $\alpha$ order is defined by

$$
{ }_{0} I_{t}^{\alpha} u(t)=\frac{1}{\Gamma(\alpha)} \int_{0}^{t}(t-\tau)^{\alpha-1} u(\tau) d \tau, \quad 0<t, 0<\alpha .
$$

Now we present our analytical schemes using the convenient Adomian series, Laplace transform, and Pade approximation. We adopt the steps in [27, 28]. Considering the following general fractional differential equations (FDEs),

$$
L[u]+N[u]=g(t),
$$

where $L[u]$ is a linear operator with respect to $u$ such as ${ }_{0}^{C} D_{t}^{\alpha} u,{ }_{0}^{C} D_{t}^{\alpha} u+u,{ }_{0}^{C} D_{t}^{\alpha} u+{ }_{0}^{C} D_{t}^{\beta} u, \ldots$, we show the following iteration schemes.

(a) Take Laplace transform $\widetilde{L}$ to both sides:

$$
\widetilde{L}[L[u]]+\widetilde{L}[N[u]]=\widetilde{L}[g(t)] .
$$

We can have iteration formula (4) through inverse of Laplace transform $\widetilde{L}^{-1}$ :

$$
u(t)=f(t)+\widetilde{L}^{-1}[\widetilde{\lambda}(s) \widetilde{L}[N[u]]],
$$

where $\tilde{\lambda}(s)$ and $f(t)$ can be determined by calculation of Laplace transform to $L[u], g(t)$. This step makes (3) in time domain equivalently defined in the Laplace domain. This idea is illustrated in the solution of differential equations [27-29]. Here the $\widetilde{\lambda}(s)$ similarly plays a role as the Lagrange multipliers in the VIM [29].

(b) Through the Picard successive approximation, we can obtain the following iteration formula:

$$
u_{n+1}=f(t)+\widetilde{L}^{-1}\left[\widetilde{\lambda}(s) \widetilde{L}\left[N\left[u_{n}\right]\right]\right] .
$$

(c) Let $u_{n}=\sum_{i=0}^{n} v_{i}$ and apply the Adomian series to expand the term $N[u]$ as $\sum_{i=0}^{\infty} A_{i}$. Then the iteration formula reads

$$
\begin{gathered}
v_{n+1}=\widetilde{L}^{-1}\left[\tilde{\lambda}(s) \widetilde{L}\left[A_{n}\right]\right], \\
v_{0}=f(t)
\end{gathered}
$$

according to [24], where $A_{i}$ is calculated by

$$
A_{i}=\frac{1}{i} \sum_{k=0}^{i-1}(k+1) v_{k+1} \frac{d A_{i-1-k}}{d v_{0}} .
$$

(d) The $n$ th-order approximation is explicitly given as

$$
u_{n}=\sum_{i=0}^{n} v_{i}
$$

The $A_{i}$ depends on the $v_{0}, v_{1}, \ldots, v_{i}$ which is denoted as

$$
A_{i}=A_{i}\left(v_{0}, v_{1}, \ldots, v_{i}\right) .
$$

This characteristic allows us to obtain the approximate solutions from $v_{0}$ and $A_{0}=N\left(v_{0}\right)$.

\section{Numerical Example and Error Analysis}

Example 1. Consider the following nonlinear FDE:

$$
{ }_{0}^{C} D_{t}^{\alpha} u+2 u^{2}=0, \quad 1<\alpha<2,
$$

with the initial conditions

$$
u(0)=1, \quad u^{\prime}(0)=0
$$

Following to the steps (a) to (d) in Section 2, we can have

$$
A_{0}=2 v_{0}^{2}
$$

where $v_{0}$ can be identified using the Laplace as $v_{0}=1$.

The iteration formula is written as

$$
\begin{gathered}
v_{n+1}=-\widetilde{L}^{-1}\left[\frac{1}{s^{\alpha}} \widetilde{L}\left[A_{n}\right]\right], \quad n \geq 0, \\
A_{0}=2 v_{0}^{2}, \quad v_{0}=1 .
\end{gathered}
$$

For $\alpha=1.9$, the Adomian series and the approximate solutions can be calculated as

$$
\begin{aligned}
& A_{1}=-4 v_{0} v_{1}, \\
& u_{1}=v_{0}+v_{1}=1-1.094478036 t^{19 / 10}, \\
& A_{2}=-2 v_{1}^{2}-4 v_{2} v_{0}, \\
& u_{2}=1-1.094478036 t^{19 / 10}+0.4484842412 t^{19 / 5},
\end{aligned}
$$

We find the new way to calculate the solution and the series is very convenient compared with the classical one. Set $n=20$ and plot the defined residual function in Figure 1:

$$
g_{n}=\log _{10}\left|{ }_{0}^{C} D_{t}^{\alpha} u_{n}+2 u_{n}^{2}\right|
$$

From Figure 2, we can conclude that iteration formula (14) and the approximate solutions are correct.

Example 2. The second example is given as

$$
{ }_{0}^{C} D_{t}^{\alpha} u=u^{3}, \quad u(0)=0, u^{\prime}(0)=1,1<\alpha<2 .
$$

We obtain the following formulae based on the Adomian series:

$$
\begin{gathered}
v_{n+1}=\widetilde{L}^{-1}\left[\frac{1}{s^{\alpha}} \widetilde{L}\left[A_{n}\right]\right], \quad n \geq 0, \\
A_{0}=v_{0}^{3}, \quad v_{0}=t .
\end{gathered}
$$




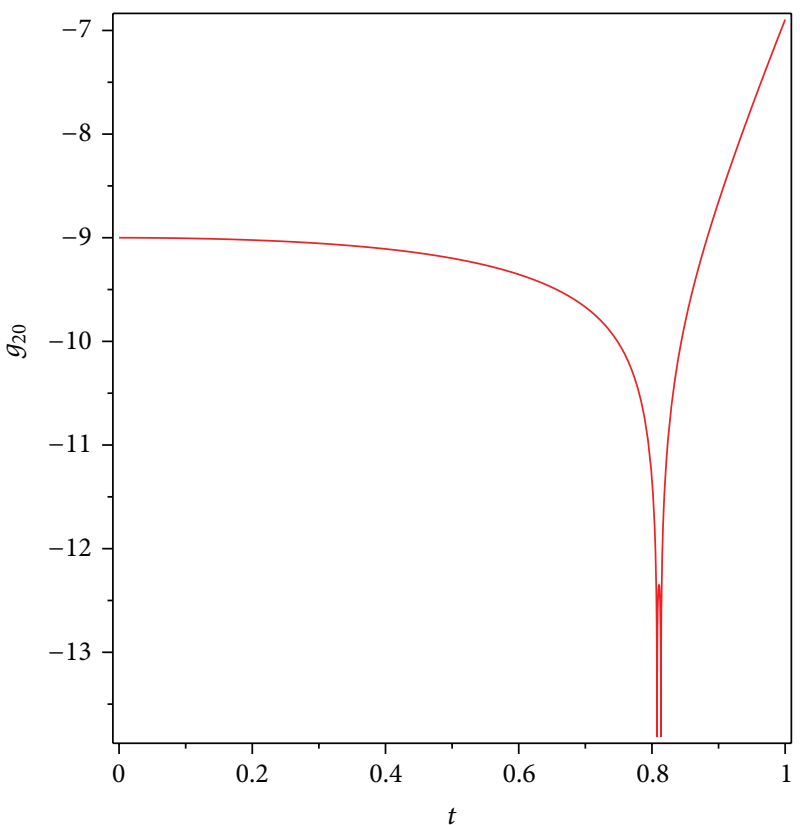

FIgURE 1: Residual function (16).

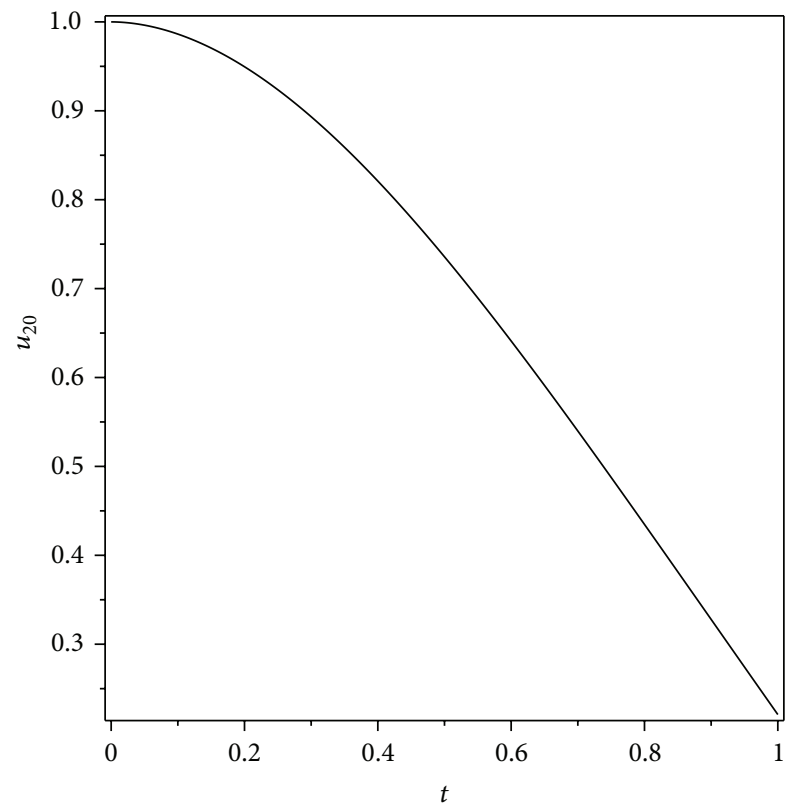

FIGURE 2: Approximate solution of (11).

For $\alpha=1.5$, we can have the approximate solutions successively as

$$
\begin{aligned}
& u_{0}=t \\
& u_{1}=t+0.1146289948 t^{9 / 2}, \\
& u_{2}=t+0.1146289948 t^{9 / 2}+0.01595982144 t^{8}
\end{aligned}
$$

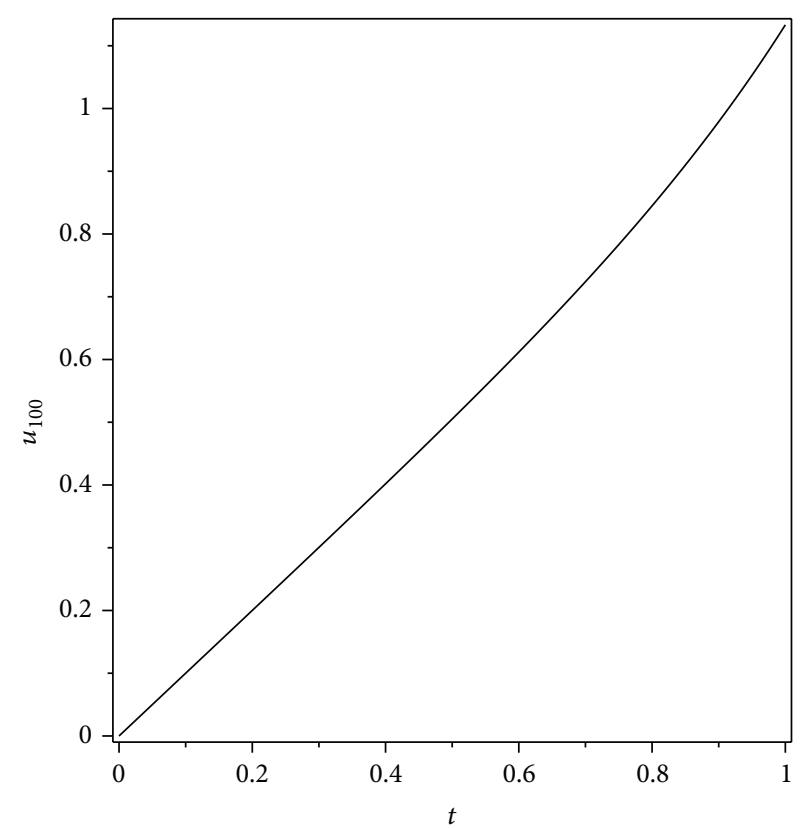

FIgURE 3: Approximate solution of (17).

$$
\begin{aligned}
u_{3}= & t+0.1146289948 t^{9 / 2}+0.01595982144 t^{8} \\
& +0.002314983909 t^{23 / 2}
\end{aligned}
$$

We plot the $u_{100}$ in Figure 3.

\section{Conclusions}

This study applied the convenient Adomian series to fractional differential equations whose order is between one and two. Two nonlinear examples are used to illustrate the basics of the steps. We found the calculation of the solutions is more convenient and more rapid compared with the classical version. The approximate order here can be chosen as $n=100$ while this choice is impossible for the classical definition. This merit is particularly nice for the fractional differential equations and the results show this purpose.

\section{Conflict of Interests}

The authors declare that there is no conflict of interests regarding the publication of this paper.

\section{Acknowledgments}

This work was financially supported by the Guangxi Natural Science Foundation (Grant no. 2013GXNSFBA019021), the Scientific Research Foundation of Guangxi University (Grant no. XBZ1205422013), and SWPU Science and Technology Fund (no. 2013XJZ029). 


\section{References}

[1] F. Mainardi, "Fractional relaxation-oscillation and fractional diffusion-wave phenomena," Chaos, Solitons and Fractals, vol. 7, no. 9, pp. 1461-1477, 1996.

[2] I. Podlubny, Fractional Differential Equations, vol. 198 of Mathematics in Science and Engineering, Academic Press, San Diego, Calif, USA, 1999.

[3] D. Baleanu and S. I. Muslih, "Lagrangian formulation of classical fields within Riemann-Liouville fractional derivatives," Physica Scripta, vol. 72, no. 2-3, pp. 119-121, 2005.

[4] K. Diethelm and N. J. Ford, "Analysis of fractional differential equations," Journal of Mathematical Analysis and Applications, vol. 265, no. 2, pp. 229-248, 2002.

[5] D. Baleanu, K. Diethelm, E. Scalas, and J. J. Trujillo, Fractional Calculus Models and Numerical Methods, vol. 3 of Series on Complexity, Nonlinearity and Chaos, World Scientific, Boston, Mass, USA, 2012.

[6] G.-C. Wu and D. Baleanu, "Discrete fractional logistic map and its chaos," Nonlinear Dynamics, vol. 75, no. 1-2, pp. 283-287, 2014.

[7] G. C. Wu and D. Baleanu, "Chaos synchronization of the discrete fractional logistic map," Signal Processing, vol. 102, pp. 96-99, 2014.

[8] M. M. Meerschaert and C. Tadjeran, "Finite difference approximations for fractional advection-dispersion flow equations," Journal of Computational and Applied Mathematics, vol. 172, no. 1, pp. 65-77, 2004.

[9] S. Shen and F. Liu, "Error analysis of an explicit finite difference approximation for the space fractional diffusion equation with insulated ends," The ANZIAM Journal: The Australian \& New Zealand Industrial and Applied Mathematics Journal, vol. 46, pp. C871-C887, 2005.

[10] H. Sun, W. Chen, C. Li, and Y. Chen, "Fractional differential models for anomalous diffusion," Physica A: Statistical Mechanics and its Applications, vol. 389, no. 14, pp. 2719-2724, 2010.

[11] K. Diethelm, N. J. Ford, and A. D. Freed, "A predictor-corrector approach for the numerical solution of fractional differential equations," Nonlinear Dynamics, vol. 29, no. 1-4, pp. 3-22, 2002.

[12] C. Yang and F. Liu, "A computationally effective predictorcorrector method for simulating fractional order dynamical control system," The ANZIAM Journal: The Australian \& New Zealand Industrial and Applied Mathematics Journal, vol. 47, pp. C168-C184, 2006.

[13] W. Deng, "Short memory principle and a predictor-corrector approach for fractional differential equations," Journal of Computational and Applied Mathematics, vol. 206, no. 1, pp. 174-188, 2007.

[14] J.-H. He, "Approximate analytical solution for seepage flow with fractional derivatives in porous media," Computer Methods in Applied Mechanics and Engineering, vol. 167, no. 1-2, pp. 57-68, 1998.

[15] J.-H. He, "Variational iteration method-a kind of non-linear analytical technique: some examples," International Journal of Non-Linear Mechanics, vol. 34, no. 4, pp. 699-708, 1999.

[16] G.-C. Wu and D. Baleanu, "Variational iteration method for the Burgers' flow with fractional derivatives-new Lagrange multipliers," Applied Mathematical Modelling: Simulation and Computation for Engineering and Environmental Systems, vol. 37, no. 9, pp. 6183-6190, 2013.

[17] D. Baleanu, G. C. Wu, and J. S. Duan, "Some analytical techniques in fractional calculus: realities and challenges," in
Discontinuity and Complexity in Nonlinear Physical Systems, J. A. Tenreiro Machado and A. C. J. Luo, Eds., Springer, 2014.

[18] V. Daftardar-Gejji and H. Jafari, "Adomian decomposition: a tool for solving a system of fractional differential equations," Journal of Mathematical Analysis and Applications, vol. 301, no. 2, pp. 508-518, 2005.

[19] S. Saha Ray and R. K. Bera, "An approximate solution of a nonlinear fractional differential equation by Adomian decomposition method," Applied Mathematics and Computation, vol. 167, no. 1, pp. 561-571, 2005.

[20] S. Momani and Z. Odibat, "Analytical solution of a timefractional Navier-Stokes equation by Adomian decomposition method," Applied Mathematics and Computation, vol. 177, no. 2, pp. 488-494, 2006.

[21] J.-S. Duan, J. An, and M. Xu, "Solution of system of fractional differential equations by Adomian decomposition method," Applied Mathematics B, vol. 22, no. 1, pp. 7-12, 2007.

[22] J.-S. Duan, "Recurrence triangle for Adomian polynomials," Applied Mathematics and Computation, vol. 216, no. 4, pp. 12351241, 2010.

[23] J.-S. Duan, "An efficient algorithm for the multivariable Adomian polynomials," Applied Mathematics and Computation, vol. 217, no. 6, pp. 2456-2467, 2010.

[24] J.-S. Duan, "Convenient analytic recurrence algorithms for the Adomian polynomials," Applied Mathematics and Computation, vol. 217, no. 13, pp. 6337-6348, 2011.

[25] J. S. Duan, R. Rach, D. Baleanu, and A. M. Wazwaz, "A review of the Adomian decomposition method and its applications to fractional differential equations," Communications in Fractional Calculus, vol. 3, no. 2, pp. 73-99, 2012.

[26] J.-S. Duan, T. Chaolu, and R. Rach, "Solutions of the initial value problem for nonlinear fractional ordinary differential equations by the Rach-Adomian-Meyers modified decomposition method," Applied Mathematics and Computation, vol. 218, no. 17, pp. 8370-8392, 2012.

[27] P.-Y. Tsai and C.-K. Chen, "An approximate analytic solution of the nonlinear Riccati differential equation," Journal of the Franklin Institute: Engineering and Applied Mathematics, vol. 347, no. 10, pp. 1850-1862, 2010.

[28] Y. Zeng, "The Laplace-Adomian-Pade technique for the ENSO model," Mathematical Problems in Engineering, vol. 2013, Article ID 954857, 4 pages, 2013.

[29] G. C. Wu, "Challenge in the variational iteration method-a new approach to identification of the Lagrange mutipliers," Journal of King Saud University-Science, vol. 25, no. 2, pp. 175-178, 2013. 


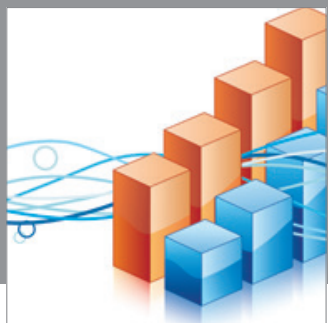

Advances in

Operations Research

mansans

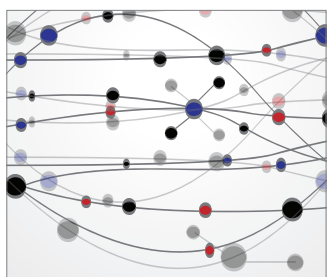

The Scientific World Journal
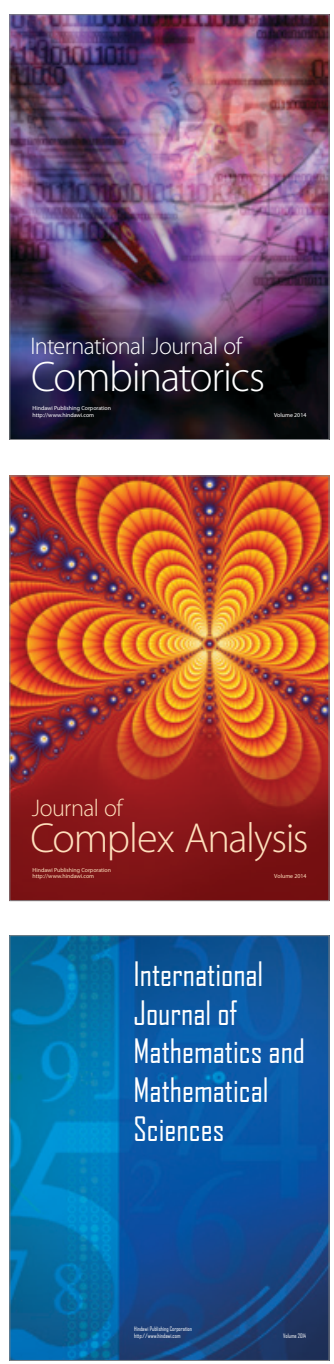
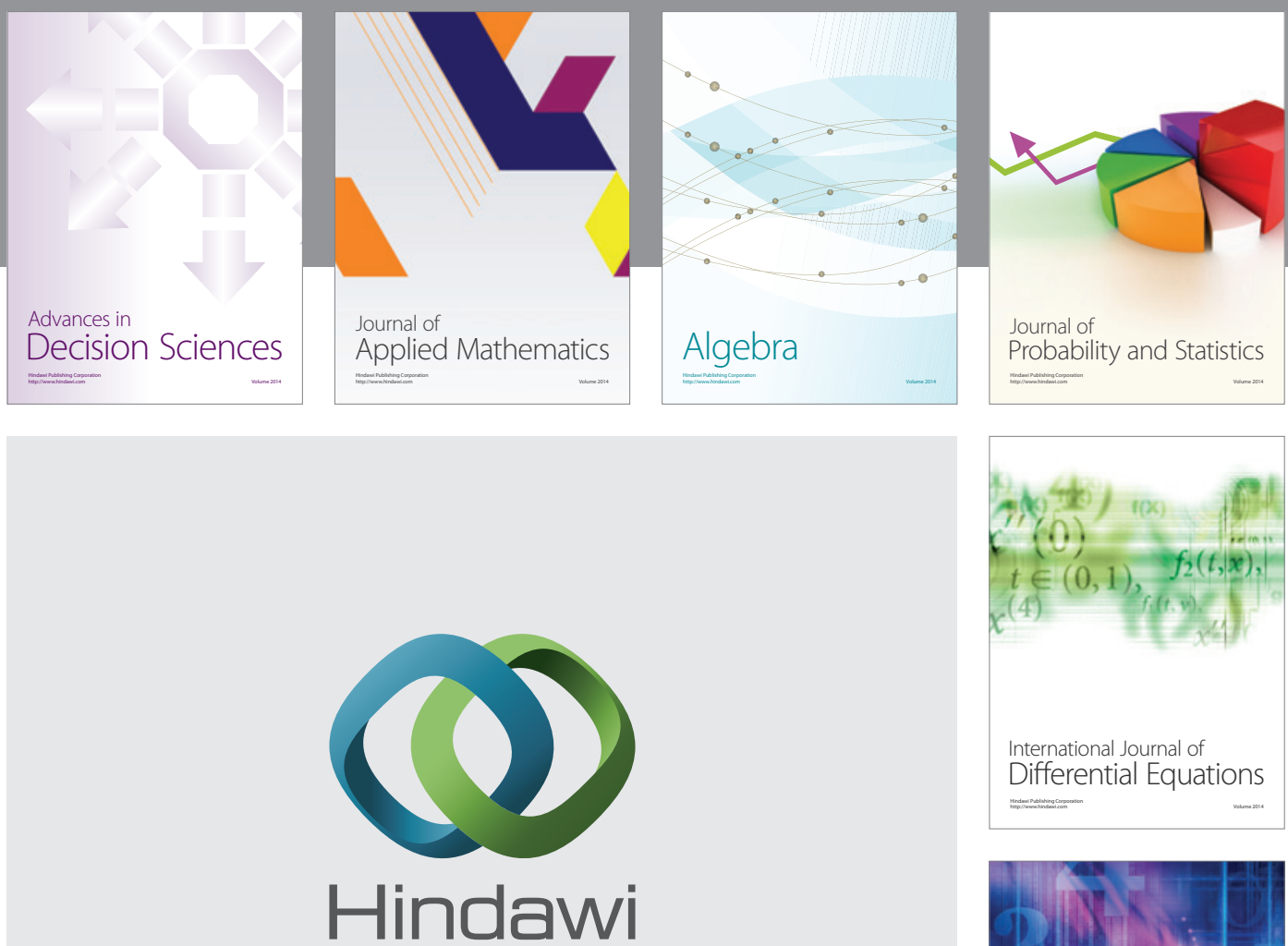

Submit your manuscripts at http://www.hindawi.com
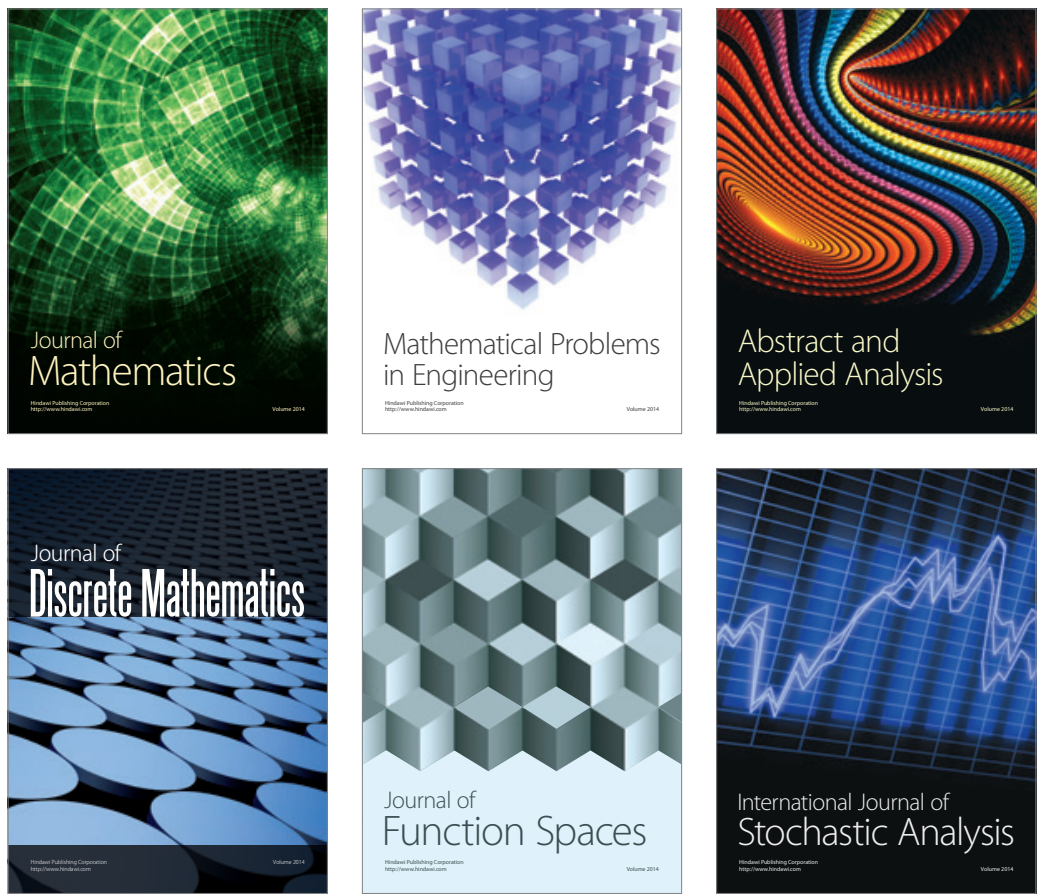

Journal of

Function Spaces

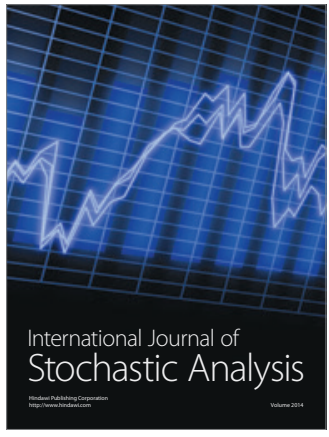

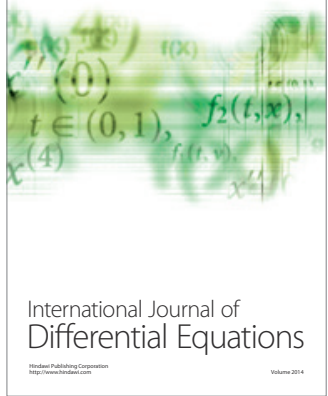
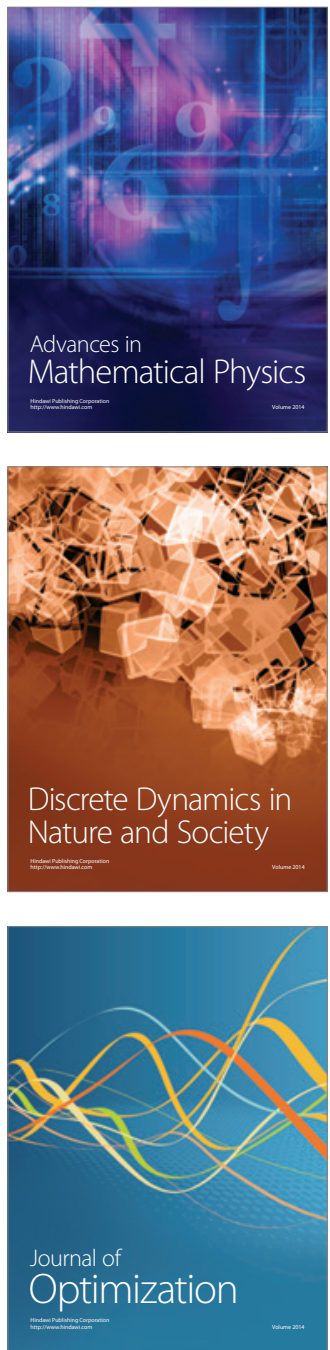